\title{
Historical relationships among areas of endemism in the tropical South America using Brooks Parsimony Analysis (BPA)
}

\author{
Mário Sérgio Sigrist ${ }^{1,3}$ \& Claudio José Barros de Carvalho ${ }^{2}$ \\ ${ }^{1}$ Departamento de Genética, Escola Superior de Agricultura Luiz de Queiroz, \\ Universidade de São Paulo - USP, \\ Av. Pádua Dias, 11, CEP 13400-970, CP 83, Piracicaba, SP, Brazil \\ ${ }^{2}$ Departamento de Zoologia, Universidade Federal do Paraná - UFPR, \\ Jardim das Américas, CEP 81531-980, CP 19020, Curitiba, PR, Brazil \\ email: cjbcarva@ufpr.br,http://zoo.bio.ufpr.br/diptera/diptera-c/index.html \\ ${ }^{3}$ Corresponding author: Mário Sérgio Sigrist, email: mariosigrist@yahoo.com.br, http://www.esalq.usp.br
}

SIGRIST, M.S. \& CARVALHO, C.J.B. Historical relationships among areas of endemism in the tropical South America using Brooks Parsimony Analysis (BPA). Biota Neotrop., 9(4): http://www.biotaneotropica.org.br/v9n4/en/abstract?article+bn02509042009.

\begin{abstract}
Areas of endemism are the smallest units of biogeographical analysis. One of its definitions is that these areas harbor organisms with restricted distributions caused by non random historical factors. The aim of this study was to examine historical relationships among areas of endemism in the Neotropics using Brooks Parsimony Analysis (BPA). We applied BPA to 12 unrelated taxa distributed within two sets of endemic areas in order to: (1) compare the proposed endemic area classifications; (2) examine whether Amazonia and Atlantic Forest are true biogeographic units and, (3) examine whether the inclusion of open area formations influence area relationships of the surrounding forests. General area cladograms revealed a basal split between Amazonian and Atlantic forests, suggesting that these areas have been isolated for a long period of time. All Atlantic forest endemic areas formed a monophyletic cluster, showing a sequence of vicariant events from north to south. The hypothesis that Amazonia is a composite area, made up of different historical units, is herein corroborated. When Cerrado and Caatinga (grasslands and savannas) are included, internal area relationships within Amazonia change, indicating that area classification schemes comprising forests and open formations should be preferred given the complementary history of these areas.

Keywords: area classification, area relationships, historical biogeography, Neotropical region.
\end{abstract}

SIGRIST, M.S. \& CARVALHO, C.J.B. Relacionamentos históricos entre áreas endêmicas na região tropical da América do Sul utilizando a Análise de Parcimônia de Brooks (BPA). Biota Neotrop., 9(4): http://www.biotaneotropica.org.br/v9n4/pt/abstract?article+bn02509042009.

Resumo: Áreas de endemismo são consideradas as menores unidades de análise biogeográfica, podendo ser definidas como regiões de concentração de organismos de distribuição restrita, gerada por fatores históricos. O presente estudo buscou examinar os relacionamentos históricos entre áreas de endemismo na região tropical da América do Sul por meio do método da Análise de Parcimônia de Brooks (BPA). Para tal, foram selecionados 12 taxa filogeneticamente distintos, distribuídos dentro de duas classificações de áreas endêmicas previamente propostas, visando: (1) comparar as classificações de áreas endêmicas; (2) examinar se a Amazônia e a Mata Atlântica são unidades biogeográficas verdadeiras; (3) avaliar se a inclusão de áreas de vegetação aberta influencia os relacionamentos entre áreas florestais vizinhas. Os cladogramas gerais de áreas revelaram uma separação basal entre as áreas Amazônicas e Atlânticas, sugerindo um longo período de isolamento. As áreas endêmicas da Mata Atlântica foram agrupadas em um único grupo, com uma seqüência de eventos vicariantes do norte em direção ao sul. A hipótese de que a Amazônia é uma área composta por unidades históricas distintas foi corroborada. A inclusão do Cerrado e Caatinga, alterou os relacionamentos internos entre áreas Amazônicas, indicando que os esquemas de classificação de áreas endêmicas que incluem tanto áreas florestais quanto abertas devem ser preferidos devido a complementaridade entre as histórias evolucionárias destas áreas.

Palavras-chave: classificação de áreas, relações entre áreas, biogeografia histórica, região Neotropical. 


\section{Introduction}

The tropical South America is well known for its remarkable biodiversity and its many regions and habitats with numerous endemic species. This diversity may be due to heterogeneity of abiotic conditions as well as a complex geological history, both responsible for the patterns of species distribution and diversification over geological time (Amorim \& Pires 1996). In fact, the spatial structure of the Neotropical biodiversity has long been studied by evolutionary biologists, particularly the ones interested in understanding the processes that explain the origin of this diversification. One of the first hypotheses for the origin of Neotropical biodiversity was the Pleistocene refugia, which was based on avian distributions and paleoclimatic data from Amazonia (Haffer 1974).

Although several aspects of this theory have been criticized, Haffer (1974) showed that the distributions of many taxa present a shared, non random distribution, which may be used to define areas of endemism. Areas of endemism are the smallest units of biogeographical analysis and could be defined as groupings of organisms with restricted distributions caused by historical factors (Harold \& Mooi 1994, Morrone 1994, Linder 2001). These areas may be especially important because they maintain unique taxa due to biodiversity production in the past and also prevent the extinction of species that were once widespread (Brooks et al. 1992). An important property of such areas is that they may be hierarchically organized, with endemic areas that share common histories grouped into biogeographic provinces, which in turn can be grouped to form biogeographic regions (Morrone 2006).

Considering the historical constraints of endemic areas and their influence on biodiversity organization, many studies attempted to delimit these areas in the tropical South America, especially in species rich regions, such as the Amazonian and the Atlantic rainforests (Cracraft 1985, Amorim \& Pires 1996, Silva \& Oren 1996, Costa et al. 2000, Silva et al. 2004, Morrone 2006, Sigrist \& Carvalho 2008). While classifications may be similar, they vary depending on the taxa of interest and the methods used. Moreover, endemic areas of some Neotropical regions, such as the grasslands or savannas, still need to be better investigated. Consequently, shortcomings have limited the interpretation of the biotic evolution in South America, since a correct delimitation of areas of endemism is essential to infer endemic area relationships (Ebach 1999).
The existence of congruent patterns among multiple taxa over endemic areas supports a common history of response to vicariant events (Cracraft 1985). In this sense, besides area definition, biogeographers are often concerned about developing hypotheses of area relationships that specify the sequence and timing of vicariance events (Marks et al. 2002). Hypothesis of area relationships within the tropical South America have been formulated by a number of studies using different approaches. Silva \& Oren (1996) and Bates (1998) performed parsimony analysis of endemicity (PAE) to infer area relationships within Amazonia, concluding that the region should be regarded as a true biogeographic unit divided in Upper and Lower Amazonia. However, the use of PAE to infer area relationships is limited due to the absence of phylogenetic information from the species and thus, it may not be considered a historical method (Humphries \& Parenti 1999). Using phylogenetic information, Cracraft \& Prum (1988) and Amorim \& Pires (1996) indicated that some Amazonian areas were more closely related to the Atlantic Forest, suggesting a historical connection between these biomes. An important weakness of these studies, however, is the non-inclusion of grasslands or savannas in the analysis, what may result in false relationships due to the shared evolutionary history between forested and non-forested areas (Costa 2003).

Three aspects might affect biogeographic conclusions regarding tropical South America: (1) divergent delimitation of endemic areas; (2) absence of non-forested areas in biogeographic analysis of the surrounding forests and; (3) the fact that most analysis does not use a testable methodology to infer area relationships. To address how these limitations may affect general patterns of area relationships, we applied a cladistic biogeography procedure (Brooks Parsimony Analysis - BPA) to phylogenetically unrelated taxa considering two sets of endemic areas: (1) forested areas within Amazonian and Atlantic forests; and (2) the previous forested areas plus the intervening open grasslands or savannas forests (Cerrado and Caatinga). The use of these two classification schemes allowed us to test whether Amazonian and Atlantic forests represent biogeographic units and how the inclusion of unrelated biomes (Cerrado and Caatinga) may influence the interpretation of forested area relationships. We then propose changes in the usual approaches to the study of areas of endemism based on the results of these analyses.

Table 1. List of taxa selected for biogeographic analysis and respective data source.

Tabela 1. Lista dos taxa selecionados para análise biogeográfica e suas respectivas referências bibliográficas.

\begin{tabular}{cll}
\hline \multicolumn{1}{c}{ Order } & \multicolumn{1}{c}{ Taxa } & \multicolumn{1}{c}{ Source } \\
\hline Araneae & Anelosimus Simon, 1891 & Agnarsson (2005) \\
& Carapoia González-Sponga, 1999 & Huber (2005) \\
Coleoptera & Hypselotropis Jekel, 1855 & Mermudes (2005) \\
& Agaporomorphus Zimmermann, 1921 & Miller (2001) \\
Squamata & Siphlophis Fitzenger, 1843 & Prudente (1998) \\
Diptera & Coenosopsia Malloch, 1924 & Michelsen (1991); Nihei \& Carvalho (2004); \\
& & Bortolanza et al. (2006) \\
& Polietina Schnabl \& Dziedzicki, 1911 & Nihei \& Carvalho (2005) \\
& Pseudoptilolepis Snyder, 1949 & Schuehli \& Carvalho (2005) \\
Hemiptera & Balacha Melichar, 1926 & Takiya \& Mejdalani (2004) \\
& Nicomia Stal, 1858 & Albertson \& Dietrich (2005) \\
Heteroptera & Serdia Stal, 1860 & Fortes \& Grazia (2005) \\
Lepidoptera & Charis Hubner, 1819 & Hall \& Harvey (2001) \\
\hline
\end{tabular}



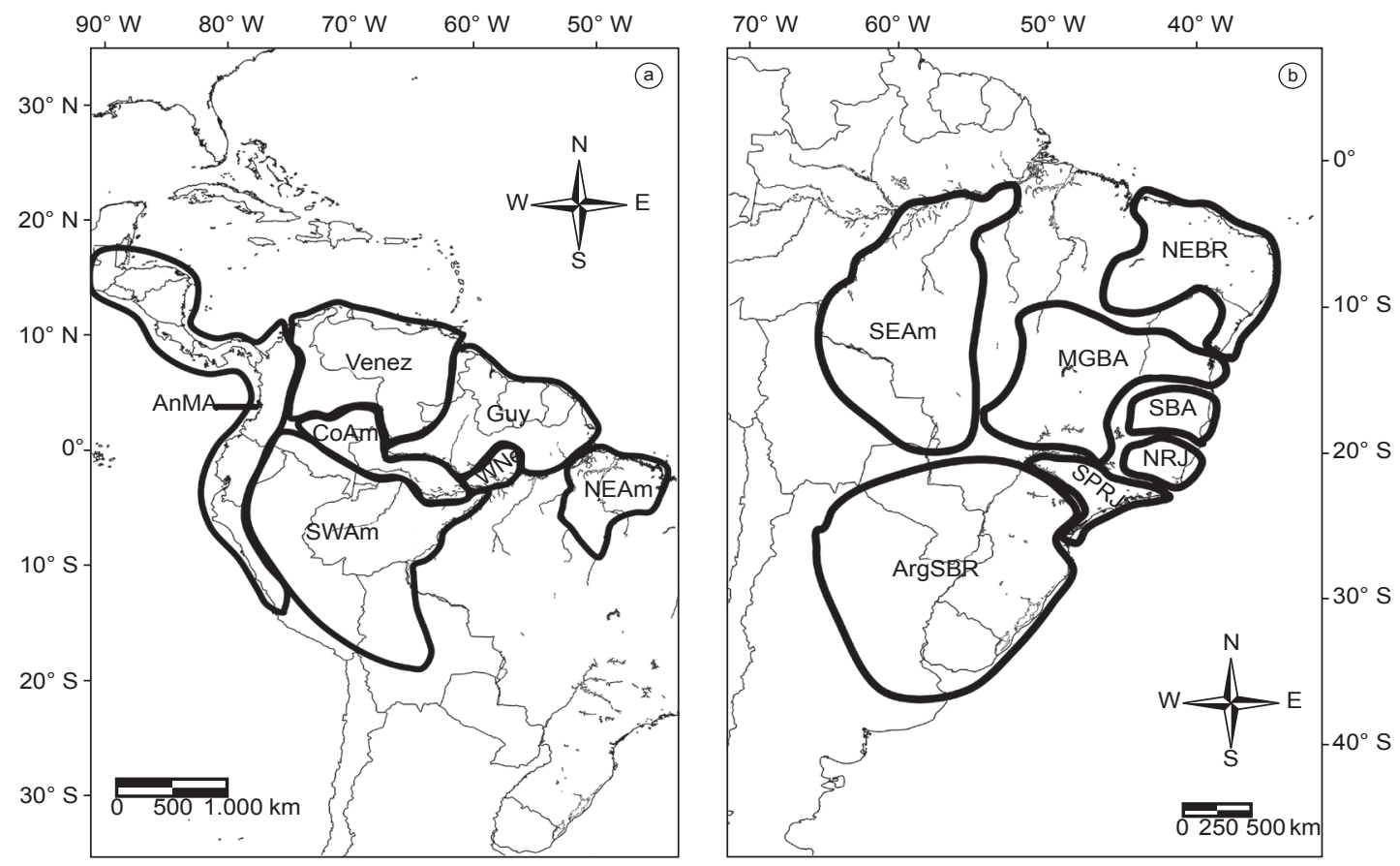

Figure 1. Endemic areas proposed in Amorim \& Pires (1996) used in primary BPA. A) Amazonian component. B) Atlantic Forest component. Note that Amazonian area SEAm was included inside Atlantic component, according to Amorim \& Pires's (1996) results. AnMA - Andes MesoAmerica; Venez - Venezuela; Guy - Guyanas; WNe - west Negro; NEAm - northeast Amazonia; SWAm - southwest Amazonia; SEAm - southeast Amazonia; NEBR - northeast Brazil; MGBA - Minas Gerais / Bahia, SBA - south Bahia; NRJ - north Rio de Janeiro; SPRJ - São Paulo / Rio de Janeiro; ArgSBR - Argentina / south Brazil.

Figura 1. Areas endêmcias propostas por Amorim \& Pires (1996) utilizadas no BPA primário. A) Componente Amazônico. B) Componente Attântico. Notar a inclusão da área amazônica SEAm dentro do componente Atlântico, conforme os resultados de Amorim \& Pires (1996). AnMA - Andes MesoAmerica; Venez - Venezuela; Guy - Guianas; WNe - oeste Negro; NEAm - nordeste Amazônia; SWAm - sudoeste Amazônia; SEAm - sudeste Amazônia; NEBR - nordeste Brasil; MGBA Minas Gerais / Bahia, SBA - sul Bahia; NRJ - norte Rio de Janeiro; SPRJ - São Paulo / Rio de Janeiro; ArgSBR - Argentina / sul Brasil.

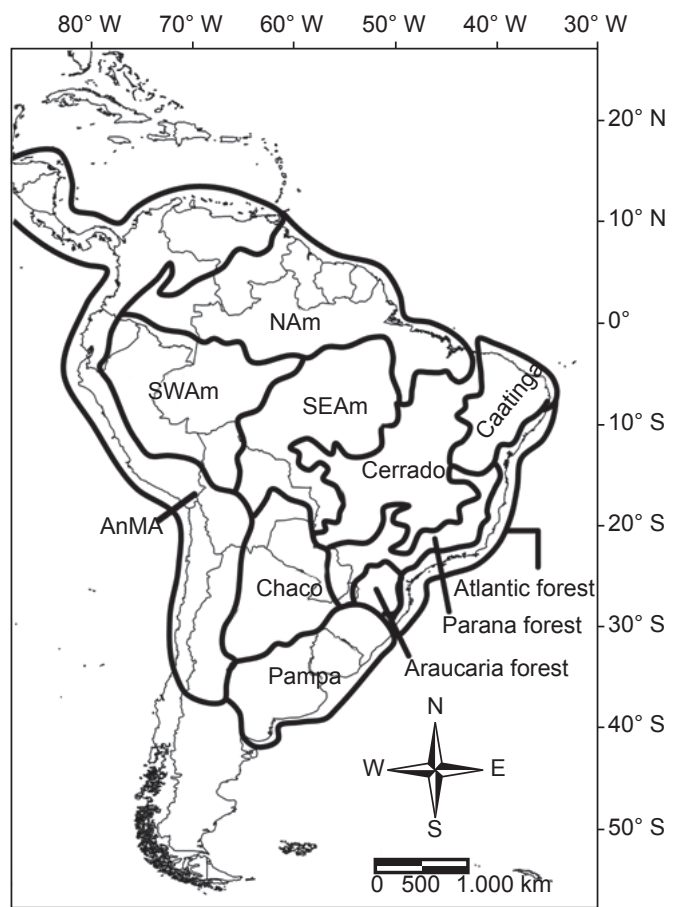

Figure 2. Endemic areas proposed in Morrone (2006), modified following Nihei \& Carvalho (2007), used in primary BPA. AnMA - Andes MesoAmerica; NAm - north Amazonia; SWAm - southwest Amazonia; SEAm - southeast Amazonia.

Figura 2. Áreas endêmicas propostas por Morrone (2006), modificadas de acordo com Nihei \& Carvalho (2007), utilizadas no BPA primário. AnMA - Andes MesoAmérica; NAm - norte Amazônia; SWAm - sudoeste Amazônia; SEAm - sudeste Amazônia.

\section{Material and Methods}

\section{Taxa analyzed}

To access general patterns of distributions and area relationships, data for phylogenetically unrelated taxa were obtained form literature based on three criteria. First, all taxa must have recent species revisions with precise details of collection localities. Second, taxa must have phylogenetic hypotheses. Third, species distributions should be restricted within tropical South America. These criteria resulted in the analysis of a total of 12 taxa comprising 114 species (Table 1).

Species distributions were assembled in a dataset and incorporated into maps for biogeographic analysis. Geographic coordinates of the species records were found using the GeoLoc tool, available at CRIA's website (http://splink.cria.org.br) and also at the Falling Rain Global Gazetteer website (www.fallingrain.com/world). The program ArcGIS 9 (ESRI 2004) was used for map elaboration and matrix construction.

\section{Areas of endemism}

Biogeographic analysis was based on two different classification schemes previously proposed for Neotropical areas of endemism. The first analysis used the areas defined in Amorim \& Pires (1996) with slight modification. We included Venezuela (Venez) because it is within the ranges of some species (Figures $1 \mathrm{a}$ and $1 \mathrm{~b}$ ). The second analysis used endemic areas proposed by Morrone (2006), with modifications following Nihei \& Carvalho (2007). Consequently, we divided the Amazonian region into three main subregions: northern Amazon (NAm), southwestern Amazon (SWAm) and southeastern Amazon (SEAm) (Figure 2). 
This division follows Amorim \& Pires (1996), in which the Amazonia, Parnaíba, Tocantins and Xingu Rivers divide northern from southern components, while the Madeira-Mamoré River divides SWAm from the SEAm.
Using these areas of endemism has two main implications. First, it allowed us to point out any possible changes in the area relationships inside Amazonia and Atlantic forest and second, we could examine whether these two main areas are true historical units or composites. (a)

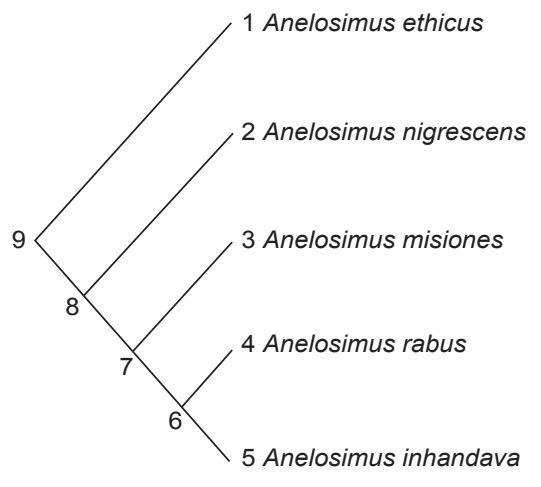

(b)

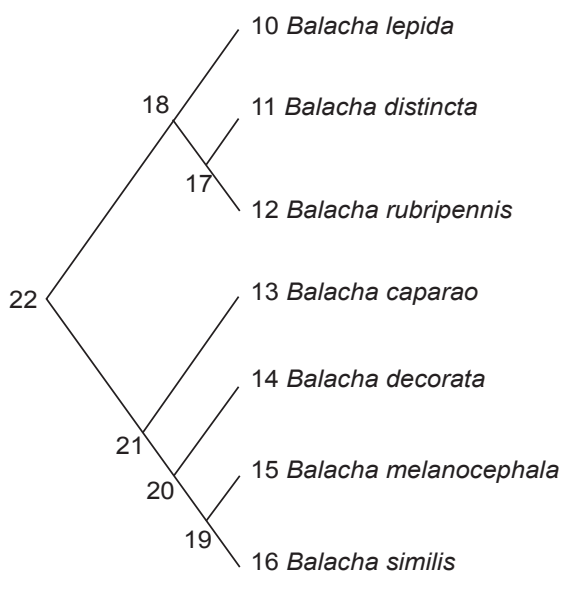

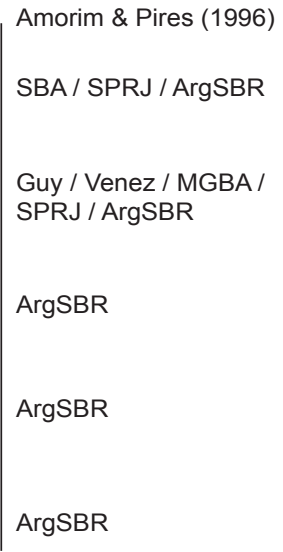

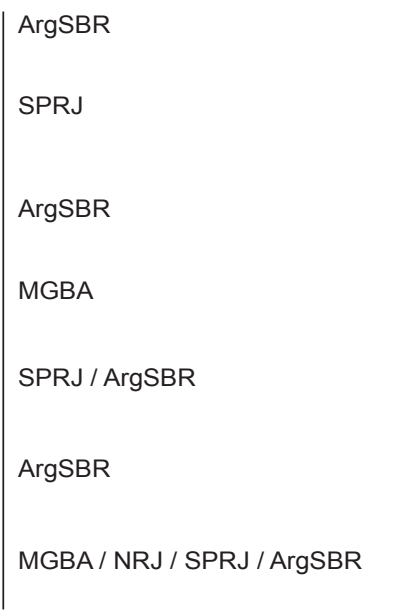

Parana forest

Parana forest

Parana forest

Parana forest / Araucária Forest / Chaco

Parana forest / Araucária Forest / Pampa / Chaco

Atlantic Forest / Parana forest / Araucária Forest
(C)

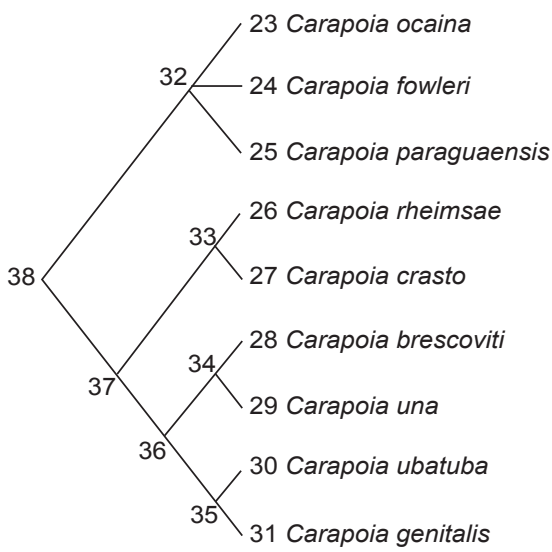

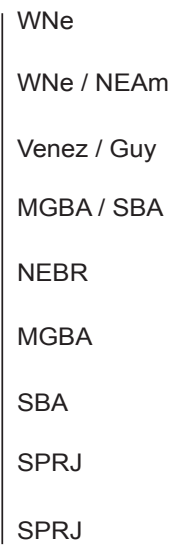

NAm
NAm
NAm
Atlantic Forest
Atlantic Forest
Atlantic Forest
Atlantic Forest
Atlantic Forest
Atlantic Forest

Figure 3. (A-M) Phylogenetic hypothesis for each taxa analyzed. Internal and terminal nodes are numbered for matrix representation. Endemic areas are shown according to the classification scheme employed. AnMA - Andes MesoAmerica; Venez - Venezuela; Guy - Guyanas; WNe - west Negro; NAm - north Amazonia; NEAm - northeast Amazonia; SWAm - southwest Amazonia; SEAm - southeast Amazonia; NEBR - northeast Brazil; MGBA - Minas Gerais / Bahia, SBA - south Bahia; NRJ - north Rio de Janeiro; SPRJ - São Paulo / Rio de Janeiro; ArgSBR - Argentina / south Brazil.

Figura 3. (A-M) Hipóteses filogenéticas para cada grupo analisado. Nós internos e ramos terminais encontram-se numerados para representação na matriz. Áreas endêmicas de cada grupo representadas de acordo com o esquema de classificação empregado. AnMA - Andes MesoAmerica; Venez - Venezuela; Guy - Guianas; WNe - oeste Negro; NAm - norte Amazônia; NEAm - nordeste Amazônia; SWAm - sudoeste Amazônia; SEAm - sudeste Amazônia; NEBR - nordeste Brasil; MGBA - Minas Gerais / Bahia, SBA - sul Bahia; NRJ - norte Rio de Janeiro; SPRJ - São Paulo / Rio de Janeiro; ArgSBR - Argentina / sul Brasil. 

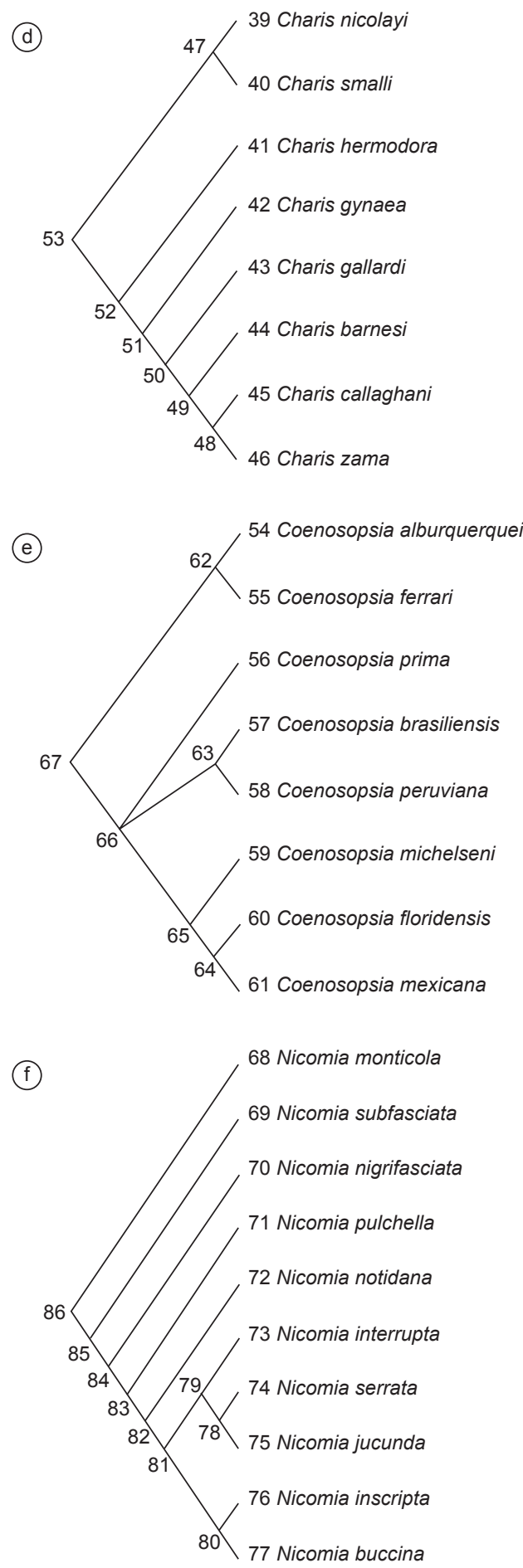

$\left\{\begin{array}{l}\text { Amorim \& Pires (1996) } \\ \text { AnMA } \\ \text { AnMA / Venez } \\ \text { MGBA / SBA / NRJ/ SPRJ } \\ \text { Guy / SWAm / WNe } \\ \text { AnMA } \\ \text { AnMA } \\ \text { Venez / SWAm / NEAm / } \\ \text { SEAm / MGBA / SPRJ }\end{array}\right.$

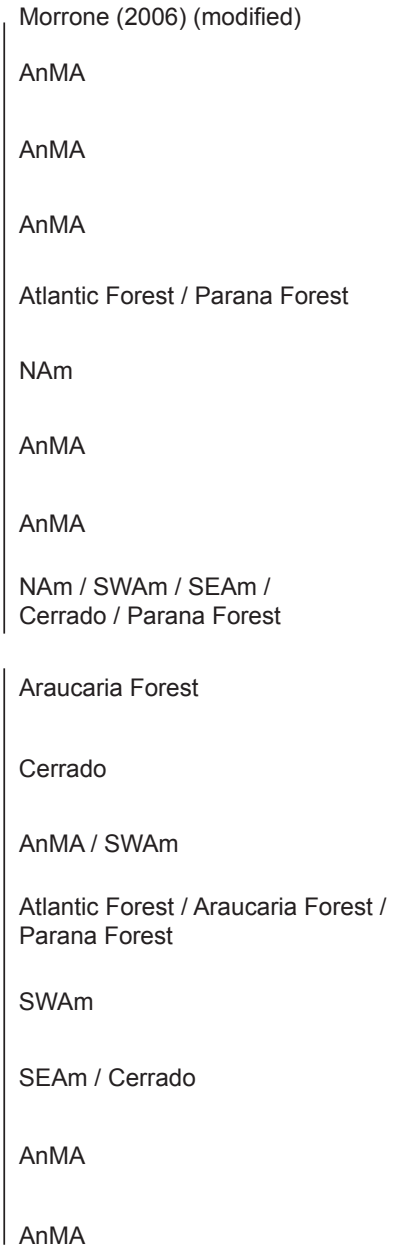

\begin{tabular}{l|l} 
SPRJ & Atlantic Forest \\
SBA / SPRJ / ArgSBR & Atlantic Forest / Araucaria Forest \\
AnMA & AnMA \\
Guy & NAm \\
CoAM & Atlantic Forest \\
SPRJ & NAm / SWAm \\
Guy / CoAM / SEAm / WNe & NAm \\
Guy / WNe & NAm \\
Guy & AnMA
\end{tabular}

Figure 3 (continued). (A-M) Phylogenetic hypothesis for each taxa analyzed. Internal and terminal nodes are numbered for matrix representation. Endemic areas are shown according to the classification scheme employed. AnMA - Andes MesoAmerica; Venez - Venezuela; Guy - Guyanas; WNe - west Negro; NAm - north Amazonia; NEAm - northeast Amazonia; SWAm - southwest Amazonia; SEAm - southeast Amazonia; NEBR - northeast Brazil; MGBA - Minas Gerais / Bahia, SBA - south Bahia; NRJ - north Rio de Janeiro; SPRJ - São Paulo / Rio de Janeiro; ArgSBR - Argentina / south Brazil.

Figura 3 (continuação). (A-M) Hipóteses filogenéticas para cada grupo analisado. Nós internos e ramos terminais encontram-se numerados para representação na matriz. Áreas endêmicas de cada grupo representadas de acordo com o esquema de classificação empregado. AnMA - Andes MesoAmerica; Venez - Venezuela; Guy - Guianas; WNe - oeste Negro; NAm - norte Amazônia; NEAm - nordeste Amazônia; SWAm - sudoeste Amazônia; SEAm - sudeste Amazônia; NEBR - nordeste Brasil; MGBA - Minas Gerais / Bahia, SBA - sul Bahia; NRJ - norte Rio de Janeiro; SPRJ - São Paulo / Rio de Janeiro; ArgSBR - Argentina / sul Brasil. 
Sigrist, M.S. \& Carvalho, C.J.B.

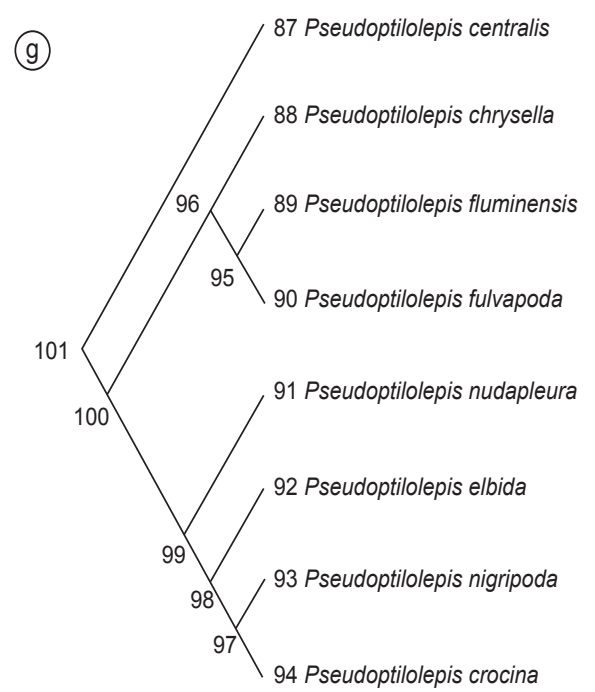

\begin{tabular}{|c|c|}
\hline Amorim \& Pires (1996) & Morrone (2006) (modified) \\
\hline AnMA & AnMA \\
\hline ArgSBR / SPRJ & Atlantic Forest / Araucaria Forest \\
\hline AnMA / SEAm / SPRJ / ArgSBR & AnMA / Atlantic Forest / Parana Forest \\
\hline AnMA / MGBA / SPRJ / ArgSBR & Atlantic Forest / Parana Forest / Araucaria Forest \\
\hline MGBA / SBA / NRJ / SPRJ / ArgSBR & Cerrado / Atlantic Forest / Parana Forest \\
\hline ArgSBR & Parana Forest \\
\hline $\begin{array}{l}\text { AnAM / SEAm / MGBA / SBA / } \\
\text { SPRJ / ArgSBR }\end{array}$ & Anma / Cerrado / Atlantic Forest / Parana Forest \\
\hline SPRJ / SBA & Atlantic Forest \\
\hline MGBA / SPRJ / ArgSBR & $\begin{array}{l}\text { Atlantic Forest / Parana Forest / } \\
\text { Araucaria Forest / Chaco }\end{array}$ \\
\hline MGBA / SPRJ / ArgSBR & Atlantic Forest / Parana Forest / Araucaria Forest \\
\hline AnMA / Venez & AnMA \\
\hline SWAm & SWAm \\
\hline AnMA / SWAm / Venez & AnMA / SWAm \\
\hline AnMA / SWAm & AnMA \\
\hline Venez & AnMA \\
\hline Guy / MGBA / SPRJ / ArgSBR & NAm / Atlantic Forest / Parana Forest \\
\hline MGBA / ArgSBR & Parana Forest / Araucaria Forest \\
\hline ArgSBR & Atlantic Forest / Araucaria Forest \\
\hline MGBA / SPRJ / ArgSBR & Atlantic Forest / Parana Forest / Araucaria Forest \\
\hline ArgSBR & Atlantic Forest \\
\hline SBA / SPRJ / ArgSBR & Atlantic Forest / Parana Forest / Araucaria Forest \\
\hline SPRJ / ArgSBR & Atlantic Forest \\
\hline MGBA / SPRJ / ArgSBR & Parana Forest / Araucaria Forest \\
\hline MGBA & Atlantic Forest \\
\hline SPRJ / ArgSBR & Atlantic Forest / Araucaria Forest \\
\hline SPRJ & Parana Forest \\
\hline
\end{tabular}

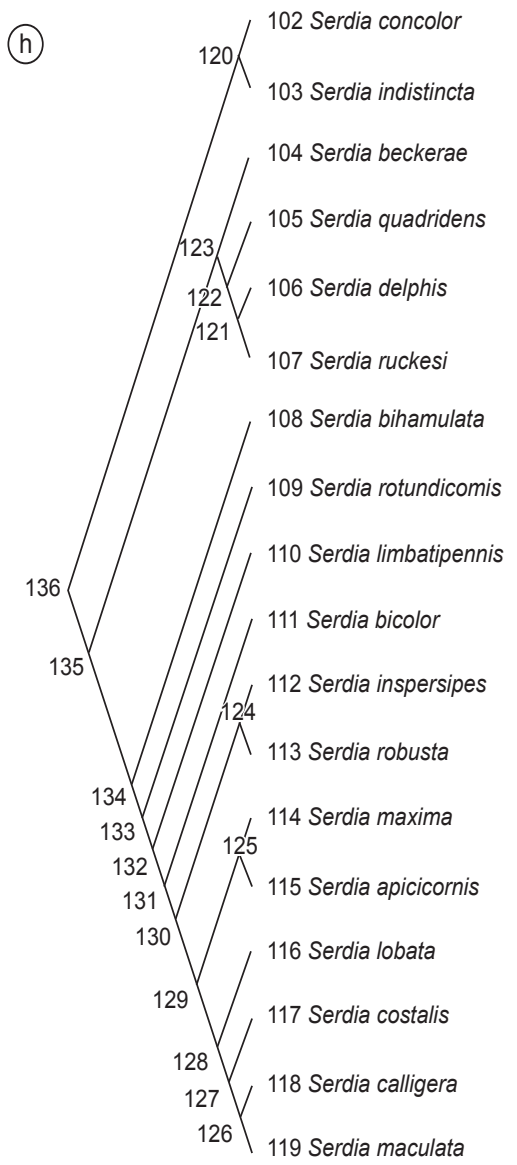

Figure 3 (continued). (A-M) Phylogenetic hypothesis for each taxa analyzed. Internal and terminal nodes are numbered for matrix representation. Endemic areas are shown according to the classification scheme employed. AnMA - Andes MesoAmerica; Venez - Venezuela; Guy - Guyanas; WNe - west Negro; NAm - north Amazonia; NEAm - northeast Amazonia; SWAm - southwest Amazonia; SEAm - southeast Amazonia; NEBR - northeast Brazil; MGBA - Minas Gerais / Bahia, SBA - south Bahia; NRJ - north Rio de Janeiro; SPRJ - São Paulo / Rio de Janeiro; ArgSBR - Argentina / south Brazil.

Figura 3 (continuação). (A-M) Hipóteses filogenéticas para cada grupo analisado. Nós internos e ramos terminais encontram-se numerados para representação na matriz. Áreas endêmicas de cada grupo representadas de acordo com o esquema de classificação empregado. AnMA - Andes MesoAmerica; Venez - Venezuela; Guy - Guianas; WNe - oeste Negro; NAm - norte Amazônia; NEAm - nordeste Amazônia; SWAm - sudoeste Amazônia; SEAm - sudeste Amazônia; NEBR - nordeste Brasil; MGBA - Minas Gerais / Bahia, SBA - sul Bahia; NRJ - norte Rio de Janeiro; SPRJ - São Paulo / Rio de Janeiro; ArgSBR - Argentina / sul Brasil. 
Area relationships in the tropical South America

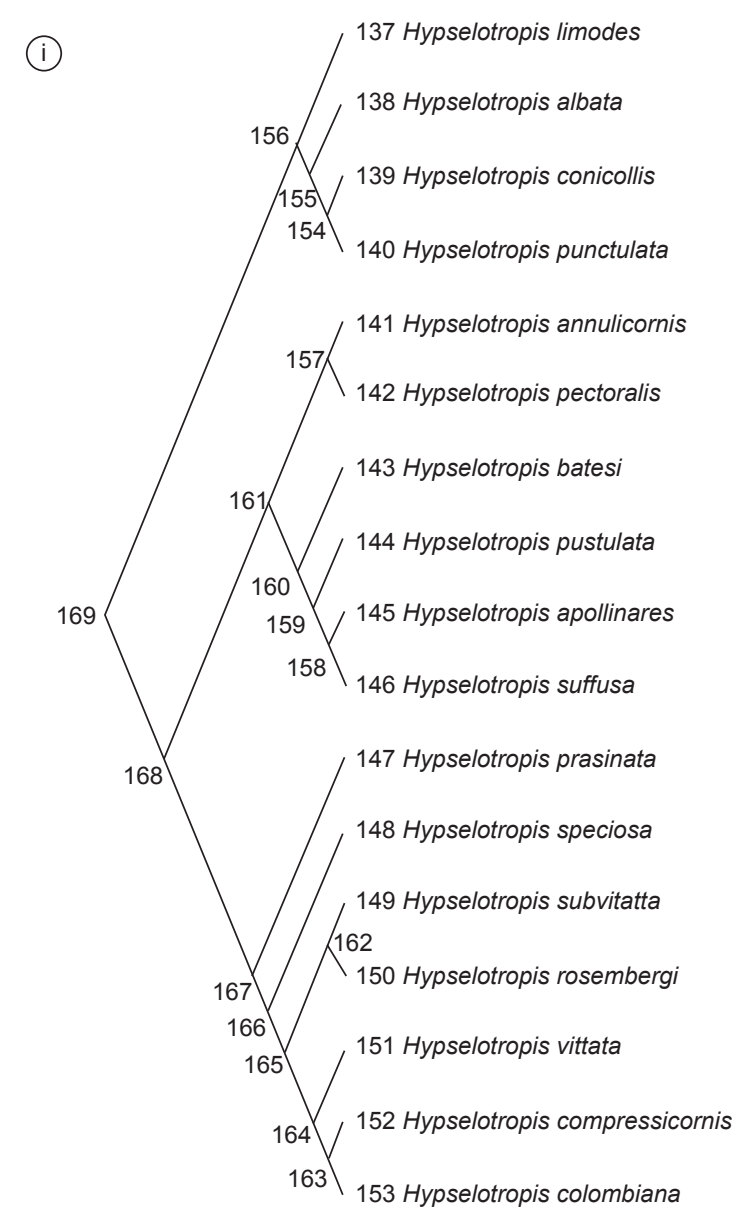

\begin{tabular}{|c|c|}
\hline Amorim \& Pires (1996) & Morrone (2006) (modified) \\
\hline SWAm & SWAm \\
\hline AnMA & AnMA \\
\hline Guy / WNe / SWAm / NEAm / SEAm & NAm / SEAm \\
\hline $\begin{array}{l}\text { SEAm / MGBA / SBA / } \\
\text { SPRJ / ArgSBR }\end{array}$ & $\begin{array}{l}\text { SEAm / Cerrado / Atlantic Forest / } \\
\text { Parana Forest }\end{array}$ \\
\hline Guy / WNe / SWAm / NEAm / SEAm & NAm / SWAm / SEAm \\
\hline SWAm & SWAm \\
\hline CoAm / SWAm / WNe / SEAm & NAm / SWAm / SEAm \\
\hline CoAm / SEAm / Guy / WNe & NAm / SEAm \\
\hline AnMA / Venez & AnMA / SWAm \\
\hline SWAm / SEAm & SWAm / SEAm \\
\hline MGBA / SBA / SPRJ / ArgSBR & Atlantic Forest / Parana Forest \\
\hline AnMA / Venez & AnMA \\
\hline AnMA & SWAm \\
\hline AnMA & AnMA \\
\hline AnMA / SWAm & SWAm \\
\hline Guy/ Venez & AnMA / NAm \\
\hline Venez & AnMA \\
\hline
\end{tabular}

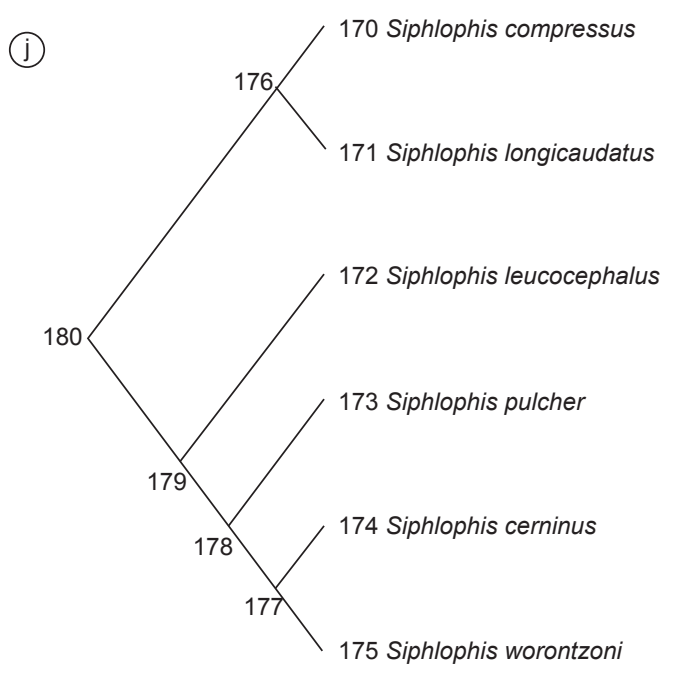

\begin{tabular}{|c|c|}
\hline $\begin{array}{l}\text { Guy / SWAm / NEAm / SEAm / } \\
\text { NEBR / SBA / SPRJ }\end{array}$ & $\begin{array}{l}\text { NAm / SWAm / SEAm / Caatinga / } \\
\text { Atlantic Forest / Parana Forest }\end{array}$ \\
\hline $\begin{array}{l}\text { NEBR / MGBA / SBA / NRJ / } \\
\text { SPRJ / ArgSBR }\end{array}$ & $\begin{array}{l}\text { Cerrado / Atlantic Forest / Parana Forest / } \\
\text { Araucaria Forest / Pampa }\end{array}$ \\
\hline MGBA / SBA & Cerrado / Atlantic Forest \\
\hline NEAm / SPRJ / ArgSBR & Atlantic Forest \\
\hline $\begin{array}{l}\text { AnMA / SWAm / Guy / WNe / } \\
\text { SEAm / ArgSBR }\end{array}$ & AnMA / NAm / SWAm / SEAm / Chaco \\
\hline SEAm / ArgSBR & SWAm / Cerrado \\
\hline
\end{tabular}

Figure 3 (continued). (A-M) Phylogenetic hypothesis for each taxa analyzed. Internal and terminal nodes are numbered for matrix representation. Endemic areas are shown according to the classification scheme employed. AnMA - Andes MesoAmerica; Venez - Venezuela; Guy - Guyanas; WNe - west Negro; NAm - north Amazonia; NEAm - northeast Amazonia; SWAm - southwest Amazonia; SEAm - southeast Amazonia; NEBR - northeast Brazil; MGBA - Minas Gerais / Bahia, SBA - south Bahia; NRJ - north Rio de Janeiro; SPRJ - São Paulo / Rio de Janeiro; ArgSBR - Argentina / south Brazil.

Figura 3 (continuação). (A-M) Hipóteses filogenéticas para cada grupo analisado. Nós internos e ramos terminais encontram-se numerados para representação na matriz. Áreas endêmicas de cada grupo representadas de acordo com o esquema de classificação empregado. AnMA - Andes MesoAmerica; Venez - Venezuela; Guy - Guianas; WNe - oeste Negro; NAm - norte Amazônia; NEAm - nordeste Amazônia; SWAm - sudoeste Amazônia; SEAm - sudeste Amazônia; NEBR - nordeste Brasil; MGBA - Minas Gerais / Bahia, SBA - sul Bahia; NRJ - norte Rio de Janeiro; SPRJ - São Paulo / Rio de Janeiro; ArgSBR - Argentina / sul Brasil. 
Sigrist, M.S. \& Carvalho, C.J.B.

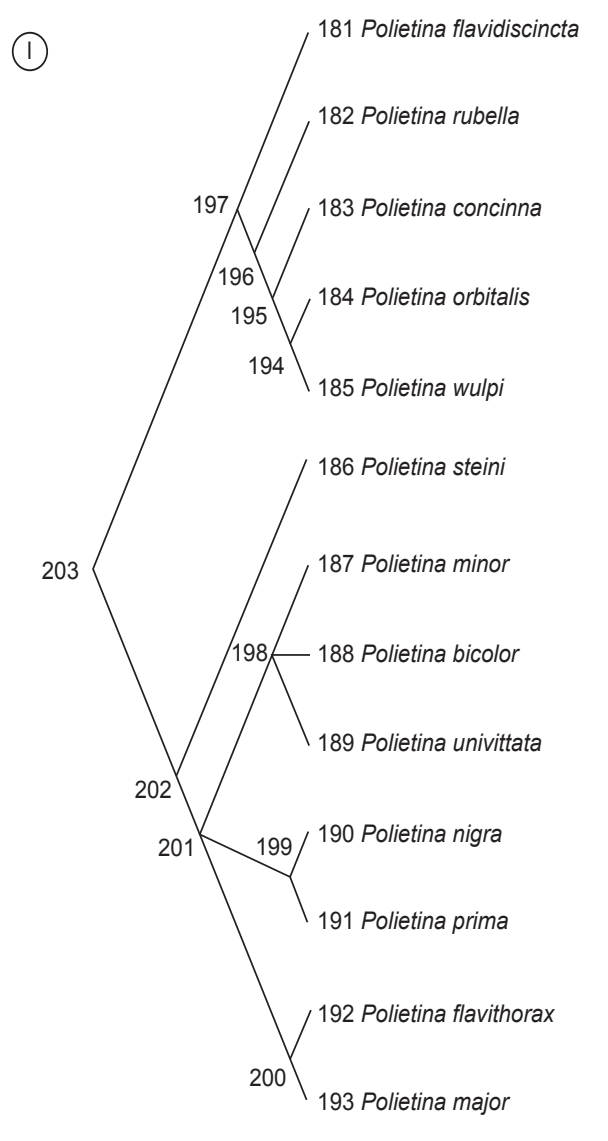

\begin{tabular}{|c|c|}
\hline Amorim \& Pires (1996) & Morrone (2006) (modified) \\
\hline SWAm / CoAm & NAm / SWAm \\
\hline AnMA & AnMA \\
\hline AnMA & AnMA \\
\hline $\begin{array}{l}\text { SWAm / SEAm / NEBR / SBA / } \\
\text { MGBA / NRJ / SPRJ / ArgSBR }\end{array}$ & $\begin{array}{l}\text { SWAm / SEAm / Cerrado / } \\
\text { Atlantic Forest / Parana Forest / } \\
\text { Araucaria Forest / Pampa }\end{array}$ \\
\hline AnMA & AnMA \\
\hline $\begin{array}{l}\text { SEAm / NEBR / SBA / } \\
\text { SPRJ / ArgSBR }\end{array}$ & $\begin{array}{l}\text { SEAm / Atlantic Forest / } \\
\text { Parana Forest / Araucaria Forest }\end{array}$ \\
\hline SPRJ / ArgSBR & Atlantic Forest / Parana Forest \\
\hline SPRJ / ArgSBR & $\begin{array}{l}\text { Atlantic Forest / Parana Forest / } \\
\text { Araucaria Forest }\end{array}$ \\
\hline SPRJ / ArgSBR & Atlantic Forest / Araucaria Forest \\
\hline $\begin{array}{l}\text { AnMA / Guy / NEAm / WNe / Venez / } \\
\text { CoAm / SEAm / NRJ / NEBR / SPRJ }\end{array}$ & $\begin{array}{l}\text { AnMA / NAm / Caatinga / } \\
\text { Atlantic Forest }\end{array}$ \\
\hline $\begin{array}{l}\text { AnMA / SWAm / SEAm / CoAm / } \\
\text { WNe / NEBR / SPRJ }\end{array}$ & NAm / SWAm / SEAm / Atlantic Forest \\
\hline $\begin{array}{l}\text { SWAm / SEAm / WNe / } \\
\text { SPRJ / ArgSBR }\end{array}$ & $\begin{array}{l}\text { NAm / SWAm / SEAm / Cerrado / } \\
\text { Atlantic Forest / Parana Forest / } \\
\text { Araucaria Forest }\end{array}$ \\
\hline $\begin{array}{l}\text { SWAm / SEAm / NRJ / } \\
\text { SPRJ / ArgSBR }\end{array}$ & $\begin{array}{l}\text { SWAm / Cerrado / Atlantic Forest / } \\
\text { Parana Forest }\end{array}$ \\
\hline
\end{tabular}
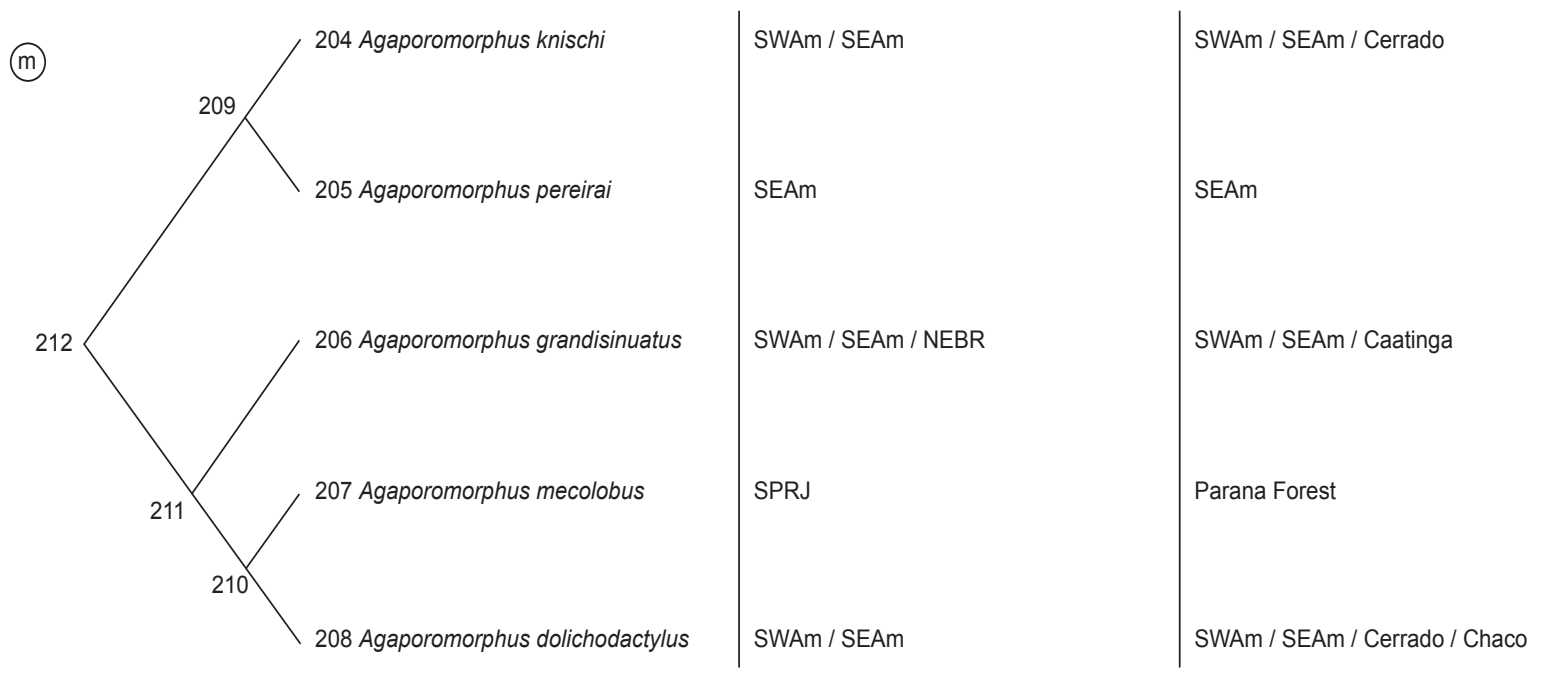

Figure 3 (continued). (A-M) Phylogenetic hypothesis for each taxa analyzed. Internal and terminal nodes are numbered for matrix representation. Endemic areas are shown according to the classification scheme employed. AnMA - Andes MesoAmerica; Venez - Venezuela; Guy - Guyanas; WNe - west Negro; NAm - north Amazonia; NEAm - northeast Amazonia; SWAm - southwest Amazonia; SEAm - southeast Amazonia; NEBR - northeast Brazil; MGBA - Minas Gerais / Bahia, SBA - south Bahia; NRJ - north Rio de Janeiro; SPRJ - São Paulo / Rio de Janeiro; ArgSBR - Argentina / south Brazil.

Figura 3 (continuação). (A-M) Hipóteses filogenéticas para cada grupo analisado. Nós internos e ramos terminais encontram-se numerados para representação na matriz. Áreas endêmicas de cada grupo representadas de acordo com o esquema de classificação empregado. AnMA - Andes MesoAmerica; Venez - Venezuela; Guy - Guianas; WNe - oeste Negro; NAm - norte Amazônia; NEAm - nordeste Amazônia; SWAm - sudoeste Amazônia; SEAm - sudeste Amazônia; NEBR - nordeste Brasil; MGBA - Minas Gerais / Bahia, SBA - sul Bahia; NRJ - norte Rio de Janeiro; SPRJ - São Paulo / Rio de Janeiro; ArgSBR - Argentina / sul Brasil. 


\section{Biogeographic analysis}

We used primary Brooks Parsimony Analysis (BPA) (Brooks 1990, Brooks \& McLennan 1991, Brooks et al. 2001), which, similar to other cladistic biogeographic methods, uses phylogenetic information of taxa to infer historical relationships among the areas where these taxa occur (VanVeller et al. 2002). BPA uses species as evolutionary markers to infer area relationships and also may be considered a co-speciation analysis (Page \& Charleston 1998). The basic procedure consists of deriving individual area cladograms for each taxa by replacing species names for the endemic areas in which they occur. Next, individual area cladograms derived from each classification scheme were summarized in a general area cladogram to establish a general pattern of area relationships. To do so, all internal and terminal nodes of phylogenetic relationships were number coded (components, Figure 3) for representation in the data matrices.

Data matrices comprising areas (rows) and components (columns) were coded for parsimony analysis (absence $=0$, presence $=1$ ). When no records exist the code is "?" to avoid a priori inferences about dispersals or extinctions (Wiley 1988). Widespread species that occurred in more than one area were treated under assumption 0 (widespread taxa as evidence for grouping areas; Humphries \& Parenti 1999). A hypothetical ancestral area coded by the absence of any components (all zero) was included to root the general cladogram (Crisci et al. 2003). The resulting data matrices were edited using the program NEXUS (Page 2001) and then exported to NONA (Goloboff 1999) for parsimony analysis. The software WINCLADA (Nixon 2002) was used for tree viewing and editing. Parsimony analysis was performed using the following commands: hold 100, hold/50, mult*100. Unsupported nodes in the resulting trees were collapsed and a strict consensus cladogram selected for discussion.

\section{Results}

Primary Brooks Parsimony Analysis using the endemic areas indicated by Amorim \& Pires (1996) resulted in two most parsimonious trees. The strict consensus cladogram consisted of 329 steps,

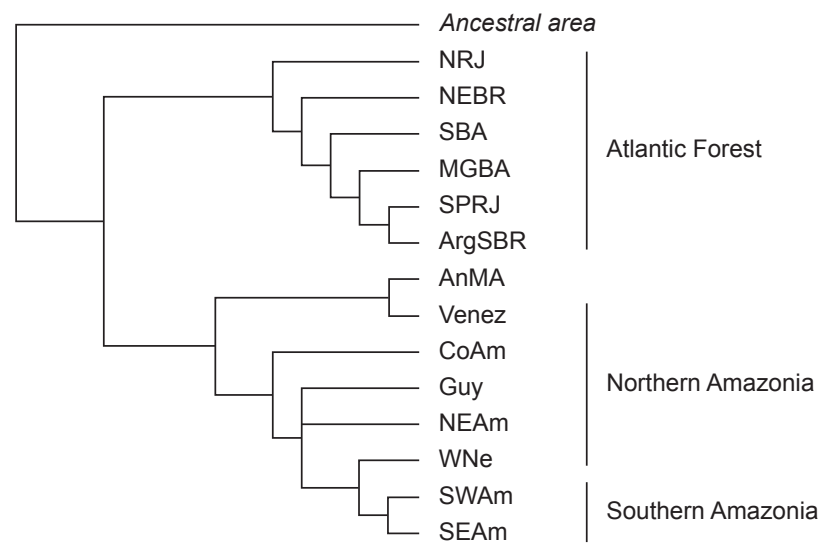

Figure 4. Strict consensus cladogram using endemic areas proposed in Amorim \& Pires (1996). AnMA - Andes MesoAmerica; Venez - Venezuela; Guy Guyanas; WNe - west Negro; NEAm - northeast Amazonia; SWAm - southwest Amazonia; SEAm - southeast Amazonia; NEBR - northeast Brazil; MGBA - Minas Gerais / Bahia, SBA - south Bahia; NRJ - north Rio de Janeiro; SPRJ - São Paulo / Rio de Janeiro; ArgSBR - Argentina / south Brazil.

Figura 4. Cladograma de consenso estrito obtido empregando áreas endêmicas propostas por Amorim \& Pires (1996). AnMA - Andes MesoAmerica; Venez Venezuela; Guy - Guianas; WNe - oeste Negro; NEAm - nordeste Amazônia; SWAm - sudoeste Amazônia; SEAm - sudeste Amazônia; NEBR - nordeste Brasil; MGBA - Minas Gerais / Bahia, SBA - sul Bahia; NRJ - norte Rio de Janeiro; SPRJ - São Paulo / Rio de Janeiro; ArgSBR - Argentina / sul Brasil.
CI: 64 and RI: 62 (Figure 4). The general area cladogram from BPA indicates a basal branching that separates Amazonian and Andean forests from Atlantic forests. In the Atlantic forests all areas clustered into a single monophyletic clade distinct from the other areas. The position of NRJ as the sister-group of the remaining Atlantic Forest component might be explained by the few taxa found in the area. For further discussion, this area may be incorporated into SPRJ, based on literature (Silva et al. 2004). Amazonian and Andean forests comprise a second main component which also has a basal dichotomy with Venez close to AnMA. Also, in the cladogram southern Amazonian area (SWAm and SEAm) are closely related and nested within Amazonian forests.

The second analysis, using the areas in Morrone (2006) with modifications following Nihei \& Carvalho (2007), resulted in three most parsimonious trees. The strict consensus cladogram consisted of 317 steps, CI: 66 and RI: 50 (Figure 5). This general cladogram shows a basal position of two open vegetation biomes, namely Pampa and Caatinga. However, interpretations regarding these two biomes should be done cautiously as they are based on the presence of few species in these areas.

All remaining areas formed two distinct components. The first component comprised the Atlantic Forest areas (Atlantic, Parana, Araucaria) without establishing any relationship between them. The second component comprised all Amazonian areas within which AnMA is nested and closely related to SWAm. Note the basal position of the endemic area SEAm, not clustering with SWAm as in previous analyses. The polytomy comprising the Atlantic and Amazonian forests, the Cerrado and Chaco masked their relationships, which remained unsettled.

Polytomic relationships in the general area cladogram might be due to the excessive number of missing taxa in data matrices, coded as '?' (McLennan \& Brooks 2002). Consequently, removing groups that are absent in most areas improves cladogram resolution by resolving politomy. To reduce or eliminate polytomies, we repeated the analysis, but only with clades found in at least six of the 11 areas. In this case, a total of seven taxa comprising 75 species was used, resulting in two most parsimonious trees summarized in a strict consensus cladogram of 210 steps, CI: 66 and RI: 61 (Figure 6). In this general area cladogram, the endemic area Atlantic forest and the

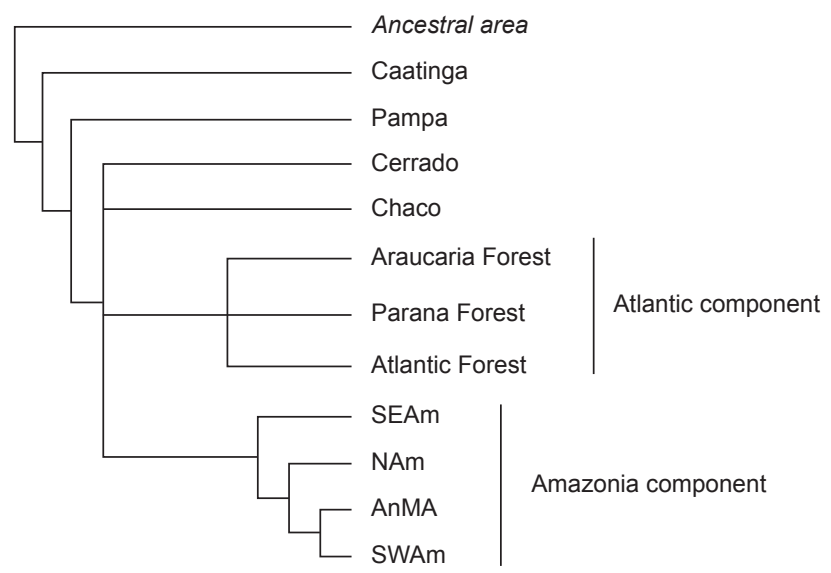

Figure 5. Strict consensus cladogram using endemic areas established in Morrone (2006), modified following Nihei \& Carvalho (2007). AnMA - Andes MesoAmerica; NAm - north Amazonia; SWAm - southwest Amazonia; SEAm - southeast Amazonia.

Figura 5. Cladograma de consenso estrito obtido empregando as áreas endêmicas estabelecidas por Morrone (2006), modificadas de acordo com Nihei \& Carvalho (2007). AnMA - Andes MesoAmérica; NAm - norte Amazônia; SWAm - sudoeste Amazônia; SEAm - sudeste Amazônia. 


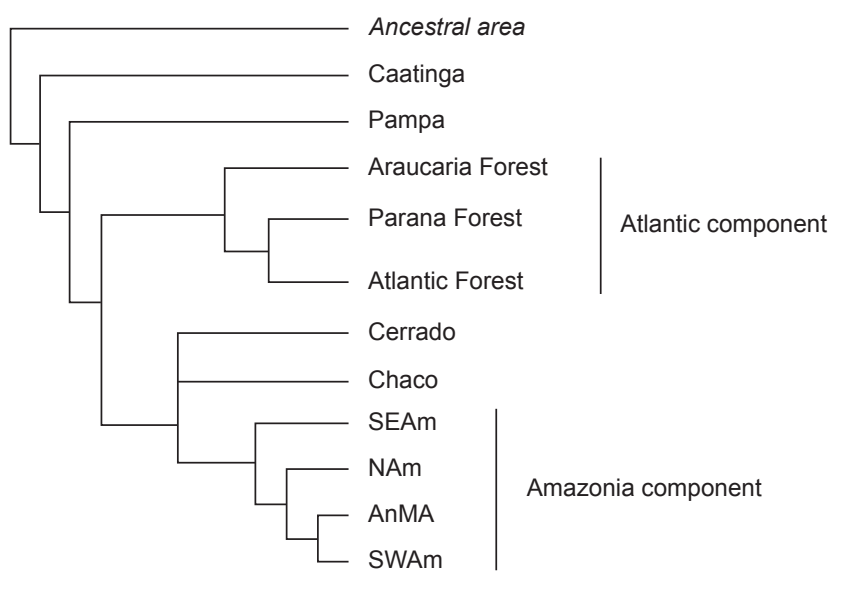

Figure 6. Strict consensus cladogram using Morrone's (2006) areas, modified accordingly to Nihei \& Carvalho (2007). In this case, only clades with species represented in at least six of the 11 areas, were considered. AnMA - Andes MesoAmerica; NAm - north Amazonia; SWAm - southwest Amazonia; SEAm - southeast Amazonia.

Figura 6. Cladograma de consenso estrito obtido a partir das áreas endêmicas propostas por Morrone (2006) modificadas de acordo com Nihei \& Carvalho (2007). Neste caso, foram considerados somente os clados com espécies representadas em pelo menos seis das 11 áreas endêmicas. AnMA - Andes MesoAmérica; NAm - norte Amazônia; SWAm - sudoeste Amazônia; SEAm - sudeste Amazônia.

Parana forest formed sister groups and the Cerrado and Chaco were clustered inside the Amazonian component, although maintaining an unresolved politomy.

\section{Discussion}

\section{Considerations about BPA}

One of the most difficult steps faced by cladistic biogeography is to summarize the information from individual area cladograms. If all individual area cladograms show a perfect congruence among their components, allopatric speciation is assumed. Indeed, this is the null hypothesis to be tested and, when different consistency indexes occur, an alternative hypothesis should be considered. This is expected, considering the intrinsic nature of the clades analyzed, coupled with the complex and cyclic influence of the geological events occurred in South America, such as marine transgressions, sea level fluctuations, climatic and topographical changes over geological time (Vanzolini 1992, Amorim \& Pires 1996).

The null hypothesis of allopatric speciation may be obscured both by widespread taxa and/or geographic paralogy (Page 1988, Nelson \& Ladiges 1996). Widespread taxa may be so because of either dispersal or lack of response to vicariant events. In this case, BPA considers widespread taxa as evidence for grouping areas (assumption 0) (Brooks et al. 2001). On the other hand, geographic paralogy occurs due to sympatry of two or more species from the same clade, as a consequence of sympatric speciation, lack of response to vicariant events or incorrect definition of areas (Ebach 1999). In order to resolve geographic paralogy, Brooks (1990) proposed a further step in BPA analysis, namely secondary BPA, characterized by the duplication of the paralogous areas found in primary BPA.

It should be noticed, however, that these two steps may be used to achieve different goals. The purpose of primary BPA is to find a general pattern of area relationships and if the null hypothesis of simple vicariance should be rejected. On the other hand, secondary BPA is used to indicate the exceptions to the general pattern (Brooks et al. 2001). Thus, we may say that primary BPA focuses on area relationships while secondary BPA focuses on understanding the processes governing taxa evolution over the areas. Considering that one aim of the present study is to infer general, wide scale patterns, we performed only primary BPA to provide a basic hypothesis of area relationships. As ambiguity was found, secondary BPA could be further conducted to explain the reticulated history of the areas. However, one limitation of secondary BPA is the complexity of analyzing large data sets (Soest \& Hadju 1997).

The efficiency of BPA as a cladistic biogeographic method has been widely debated in literature (Ebach \& Humphries 2002, Van Veller et al. 2002, Ebach et al. 2003). In fact, this debate regards not only BPA but also all methods currently employed in cladistic biogeography, since they usually give contrasting results. One problem commonly attributed to primary BPA, as here used, is that the method does not reduce paralogy and interprets ambiguity as congruence, leading to biased area relationships (Ebach \& Humphries 2002). Indeed, this is a shortcoming of primary BPA and consequently, area relationships here presented should be considered as a preliminary hypothesis that can be further tested and compared to other studies using different procedures. In addition, the other aim of this study, which was to address the problems of endemic areas classification schemes, should not have been affected by this shortcoming, since the same method was used in both analyses.

\section{Endemic area relationships}

The Amazon and the Atlantic forests are two of the most diverse regions in South America and have been the focus of many studies aiming to: (1) identify endemic areas and (2) hypothesize about the historical relationships among these areas. It is important to notice that to uncover reliable endemic areas relationship we first need to methodologically define the limits of these areas (Sigrist \& Carvalho 2008). Our results support this statement, since some area relationships changed according to the classification scheme empirically proposed. Although these differences did not allow us to chose which general cladograms furnishes a better picture of the biotic evolution in the region, areas that appeared as composites in both analysis strongly suggests that the definition of such areas should be reviewed.

In general area cladograms of the Atlantic forest, both classificatory schemes of endemic areas gave the same results: all areas formed a monophyletic area clade, yet the two schemes proposed different delimitation of the areas. Note that Amorim \& Pires (1996) divided the Atlantic component in latitudinal areas while Morrone (2006) considered a subdivision which resembles more an ecological division. Nevertheless, the results here suggest that the Atlantic forest component should be regarded as a biogeograpical unit, regardless of the classification and both with and without the inclusion of Cerrado and Caatinga. This suggestion contrasts with previous hypothesis about the hybrid nature of the Atlantic forest (Cracraft \& Prum 1988, Costa 2003).

Although Amorim \& Pires (1996) also concluded that the Atlantic forest areas form a natural assemblage, their internal relationships differ from those described here. They suggest that the Atlantic Forest may be divided into northern and southern components at the valley of the Paraíba do Sul River between the States of Rio de Janeiro and Espirito Santo. In our cladogram, excluding the underestimated position of area NRJ due to the scarcity of species analyzed, area relationships support evidence for a sequence of area disjunctions by vicariant events from north to south, in a pattern similar to the one found for other groups (Rocha et al. 2005). Moreover, assuming a plausible congruence between Morrone's (2006) classification and classifications based on ecological similarities, we may conclude that 
Atlantic Dense Rainforest is more closely related to Atlantic Seasonal Forest than to Atlantic Mixed Rain Forest.

On the other hand, several studies whose goal was to clarify area relationships within Amazonia had contradictory results. Evidence corroborating the hypothesis of a composite Amazonia is well demonstrated in phylogentic studies (Cracraft \& Prum 1988, Camargo 1996, Amorim \& Pires 1996). However, other analyses employing Parsimony Analysis of Endemicity (PAE) showed the region as a historical unit, although the efficacy of this method to infer area relationships is still being debated in literature (Silva \& Oren 1996, Porzecanski \& Cracraft 2005, Nihei 2006).

Our results from primary BPA strongly support that Amazonia is a hybrid area, comprising different historical units. The area cladogram following Amorim \& Pires's (1996) classification, suggests that Venez is more related to the Andes and Central America rather than to areas of Amazonia. This composite hypothesis is different from the one presented by Amorim \& Pires (1996), since here the endemic areas SWAm and SEAm were closely related, showing a main disjunction separating northern and southern Amazonia. One of the main arguments supporting a composite Amazonia (as in Amorim \& Pires 1996) was due to the position of SEAm within the clade of Atlantic forests. According to the authors, this pattern may have been due to marine transgressions in the late Cretaceous that included parts of the Amazonas, Madeira and Mamoré rivers. However, considering the position of southeast Amazonia (SEAm) in the area cladogram here presented and the basal split between Amazonian and Atlantic forests, a different vicariant event may be responsible for the pattern - for example, the increasing dryness and formation of the Cerrado during the Tertiary (Colli 2005). If so, tests with other different classification scheme should reinforce this hypothesis.

Although Amazonian area relationships using Morrone's (2006) classification differs from those in Amorim \& Pires (1996), once again SEAm was clustered within Amazonia. It is interesting to note that including Cerrado and Caatinga changes the internal relationships of Amazonian areas, since both southern areas previously grouped are now separated. In this analysis, SWAm is a sister area of the Andean and Central America regions, thereby also supporting the hypothesis for the composite nature of Amazonia (also see Camargo 1996).

The inclusion of Cerrado and Caatinga to find reliable relationships among adjacent forested areas was previously suggested by Costa (2003). Accordingly, the Brazilian Cerrado is complementary to both Amazon and Atlantic forests because many Atlantic and Amazon taxa occur within riverside gallery forests in the Cerrado (Silva 1996). Consequently, many taxa considered to be from Cerrado are, in fact, forest species that inhabits gallery forests. One way to address this signal in biogeographical analysis is to consider the ecological requirements of the species analyzed. However such information is scarce for most Neotropical species, including the ones here analyzed.

Considering the complementary history among tropical biomes in South America and based on the two classification schemes here tested, we suggest that a mixed scheme using both Amorim \& Pires's (1996) areas of forest endemism and Morrone's (2006) non-forested areas, will probably find more consistent results. It should be noticed, however, that the delimitation of these areas can be further improved. Even though most areas proposed by Amorim \& Pires (1996) have been corroborated by other studies using different taxa, it is desirable to use a testable methodology, applied to as many taxa as possible, to define endemic areas (i.e. Parsimony Analysis of Endemicity - PAE). In addition, the use of such methods may also propose further subdivision of the Cerrado and Caatinga biomes into smaller and better defined areas.
In view of endemic area relationships, current literature has reported strongly divergent general area cladograms (Costa 2003). Besides wrong area definitions, different area relationships might be the result of using subjective methods to summarize area cladograms. To test this assumption, our hypothesis of area relationships based on primary BPA should be compared to future studies using other biogeographical methods. Although a single network of Neotropical area relationships is not likely due to dispersal and different responses of species to environmental changes, general area cladograms obtained from testable approaches may provide a general framework to better understand the biotic evolution in the tropical South America.

\section{Acknowledgements}

We thank Elaine Della Soares and Peter Lowenberg-Neto for the critical reading of the manuscript and James J. Roper for English revision. Conselho Nacional de Desenvolvimento Cientifico e Tecnológico $(\mathrm{CNPq})$ provided financial support for this PIBIC project and also to CJBC (process 302454-2005-5).

\section{References}

AGNARSSON, I. 2005. Revision and phylogenetic analysis of American Ethicus and Rupununi groups of Anelosimus (Araneae, Theridiidae). Zoologica Scripta. 34(4):389-413

ALBERTSON, J.L. \& DIETRICH, C.H. 2005. Systematics and phylogeny of the Neotropical treehopper subfamily Nicomiinae (Hemiptera, Membracidae). Revista Brasileira de Zoologia 22(1):231-283.

AMORIM, D.S. \& PIRES, M.R.S. 1996. Neotropical biogeography and a method for maximum biodiversity estimation. In: Biodiversity in Brazil, a first approach. (C.E.M. Bicudo \& N.A. Menezes, eds.). CNPq, São Paulo, p. 183-219.

BATES, J.M., HACKETT, S.J. \& CRACRAFT, J. 1998. Area-relationships in the Neotropical lowlands: an hypothesis based on raw distributions of passerine birds. Journal of Biogeography 25(4):783-793.

BORTOLANZA, M., CARVALHO, C.J.B. \& LARA, A.P.C. 2006. A new species of Coenosopsia Malloch (Diptera, Anthomyiidae) from southern Brazil and a reappraisal of the cladistic relationships of the genus. Zootaxa. 1242:37-52.

BROOKS, D.R. 1990. Parsimony analysis in historical biogeography and coevolution: Methodological and theoretical update. Systematic Zoology 39(1):14-30.

BROOKS, D.R. \& MCLENNAN, D. 1991. Phylogeny, ecology and behavior: a research program in comparative biology. University of Chicago Press, Chicago, 434 p.

BROOKS, D.R., MAYDEN, R.L. \& MCLENNAN, D. 1992. Phylogeny and biodiversity: conserving our evolutionary legacy. Trends in Ecology and Evolution 7(2):55-59.

BROOKS, D.R., VAN VELLER, M.G. P. \& MCLENNAN, D. 2001. How to do BPA, really. Journal of Biogeography 28(3):345-358.

CAMARGO, J.M. 1996. Meliponini neotropicais (Apinae, Apidae, Hymenoptera): Biogeografia histórica. Anais do II Encontro sobre Abelhas: 107-121.

COLLI, G.R. 2005. As origens e diversificação da herpetofauna do Cerrado. In Cerrado: ecologia, biodiversidade e conservação (A. Scariot, J.C.S. Silva \& J.M. Felfili, eds.). Ministério do Meio Ambiente, Brasília, p. 247-264.

COSTA, L.P. 2003. The historical bridge between the Amazon and the Atlantic Forest of Brazil: a study of molecular phylogeography with small mammals. Journal of Biogeography 30(1):71-86.

COSTA, L.P., LEITE, Y.R.L., FONSECA, G.A.B. \& FONSECA, M.T. 2000. Biogeography of South American forest mammals: endemism and diversity in the Atlantic Forest. Biotropica 32(4b):872-881.

CRACRAFT, J. 1985. Historical biogeography and patterns of differentiation within the South American avifauna: areas of endemism. Ornithological Monographs 36:49-84. 
CRACRAFT, J. \& PRUM, R.O. 1988. Patterns and processes of diversification: speciation and historical congruence in some Neotropical birds. Evolution 42(3):603-620.

CRISCI, J.V., KATINAS, L. \& POSADAS, P. 2003. Historical biogeography: an introduction. Harvard University Press, Cambridge.

EBACH, M.C. 1999. Paralogy and the centre of origin concept. Cladistics. 15(4):387-391.

EBACH, M.C., HUMPHRIES C.J. \& WILLIAMS D.M. 2003. Phylogenetic biogeography deconstructed. Journal of Biogeography 30(9):1285-1296.

EBACH, M.C. \& HUMPHRIES, C.J. 2002. Cladistic biogeography and the art of discovery. Journal of Biogeography 29(4):427-444.

Environmental Systems Research Institute - ESRI. 2004. ArcGIS 9. ESRI, New York.

FORTES, N.D.F. \& GRAZIA, J. 2005. Revisão e análise cladística de Serdia Stal (Heteroptera, Pentatomidae, Pentatomini). Revista Brasileira de Entomologia 49(3):294-339.

GOLOBOFF, P. 1999. NONA (No Name) ver. 2.0. P. Goloboff, Tucumán.

HAFFER, J. 1974. Avian speciation in tropical South America. Nuttall Ornithology Club, Cambridge.

HALL, J.P.W. \& HARVEY, D.J. 2001. Phylogenetic revision of the Charis gynaea group (Lepidoptera: Riodinidae) with comments on historical relationships among Neotropical areas of endemism. Annals of the Entomological Society of America 94(5):631-647.

HAROLD, A.S. \& MOOI, M.D. 1994. Areas of endemism: definition and recognition criteria. Systematic Biology 43(2):261-266.

HUBER, B.A. 2005. Revision and cladistic analysis of the spider genus Carapoia González-Sponga (Araneae: Pholcidae), with descriptions of the new species from Brazil's Atlantic Forest. Invertebrate Systematics 19(6):541-556.

HUMPHRIES, C.J. \& PARENTI, L.R. 1999. Cladistic Biogeography. 2 ed. Oxford University Press, Oxford. (Oxford Biogeohraphic Series 12.)

LINDER, H.P. 2001. On areas of endemism, with an example from the african Restionaceae. Syst. Biol. 50(6):892-912.

MARKS, B.D., HACKETT, S.J. \& CAPPARELLA, A.P. 2002. Historical relationships among Neotropical lowland forest areas of endemism as determined by mitochondrial DNA sequence variation within the Wedgebilled Woodcreeper (Aves: Dendrocolaptidae: Glyphorynchus spirurus). Molecular Phylogenetics and Evolution 24(1):153-167.

MCLENNAN, D. \& BROOKS, D.R. 2002. Complex histories of speciation and dispersal in communities: a re-analysis of some Australian bird data using BPA. Journal of Biogeography 29(8):1055-1066.

MERMUDES, J.R.M. 2005. Revisão sistemática, análise cladística e biogeografia dos gêneros Tribotropis e Hypselotropis (Coleoptera, Anthribidae, Anthribinae, Ptychoderini). Revista Brasileira de Entomologia 49(4):465-511.

MICHELSEN, V. 1991. Revision of the aberrant New World genus Coenosopsia (Diptera: Antomyiidae), with a discussion of the anthomyiid relationships. Systematic Entomology 16(1):85-104.

MILLER, K.B. 2001. Revision of the genus Agaporomorphus Zimmermann (Coleoptera: Dytiscidae). Annals of the Entomological Society of America 94(4):520-529.

MORRONE, J.J. 1994. On the identification of areas of endemism. Systematic Biology 43(3):438-441.

MORRONE, J.J. 2006. Biogeographic areas and transition zones of Latin America and the Caribbean islands based on panbiogeographic and cladistic analysis of the entomofauna. Annual Review of Entomology 51:467-494.

MORRONE, J.J. \& CARPENTER, J.M. 1994. In search of a method for cladistic biogeography: An empirical comparison of component analysis, Brooks parsimony analysis, and three-area statements. Cladistics. 10(2):99-153.

NIHEI, S.S. 2006. Misconceptions about parsimony analysis of endemicity. Journal of Biogeography 33(12):2099-2106.
NIHEI, S.S. \& CARVALHO, C.J.B. 2004. Taxonomy, cladistics and biogeography of Coenosopsia Malloch (Diptera, Anthomyiidae) and its significance to the evolution of the anthomyiids in the Neotropics. Systematic Entomology 29(2):260-275.

NIHEI, S.S. \& CARVALHO, C.J.B. 2005. Distributional patterns of the Neotropical fly genus Polietina Schnabl \& Dziedzicki (Diptera, Muscidae): a phylogeny-supported analysis using panbiogeographic tools. Papéis Avulsos em Zoologia 45(25):313-326.

NIHEI, S.S. \& CARVALHO, C.J.B. 2007. Systematics and biogeography of Polietina Schnabl \& Dziedzicki (Diptera, Muscidae): neotropical areas relationships and Amazonia as a composite area. Systematic Entomology 32(3):477-501.

NIXON, K.C. 2002. WinClada versão 1.0. K.C. NIXON, Ithaca.

PAGE, R.D.M. 1988. Quantitative cladistic biogeography: constructing and comparing area cladograms. Systematic Zoology 37(3):254-270.

PAGE, R.D.M. 2001. Nexus data editor 0.5.0. Program distributed by the author, Institute of Biomedical and Life Sciences. Univ. of Glasgow, Glasgow. Available at: http://taxonomy.zoology.gla.ac.uk/rod/NDE/nde. html (June $15^{\text {th }}$ 2009).

PAGE, R.D.M. \& CHARLESTON, M.A. 1998. Trees within trees: phylogeny and historical associations. Trends in Ecology and Evolution 13(9):356-359.

PORZECANSKI, A.L. \& CRACRAFT, J. 2005. Cladistic Analysis of distributions and endemism (CADE): using raw distributions of birds to unravel the biogeography of South American aridlands. Journal of Biogeography 32(2):261-275.

PRUDENTE, A.L.C. 1998. Revisão, filogenia e alimentação de Siphlophis Fitzinger, 1843 (Serpentes, Colubridae, Xenodontinae, Pseudoboini). Tese de Doutorado, Universidade Federal do Paraná, Paraná, 165 p.

ROCHA, R.P., SILVA, M.B. \& BRAGAGNOLO, C. 2005. Faunistic similarities and historical biogeography of the Harvestman of southern and southwestern Atlantic Forest of Brazil. Journal of Arachnology 33(2):290-299.

SCHUEHLI, G.S. \& CARVALHO, C.J.B. 2005. Revision and cladistics of the Neotropical genus Pseudoptilolepis Snyder (Diptera, Muscidae). Revista Brasileira de Zoologia 22(1): 23-34.

SILVA, J.M.C. 1996. Distribution of Amazonian and Atlantic birds in gallery forests of the cerrado region, South America. Ornitologia Neotropical $7(1): 1-18$.

SILVA, J.M.C. \& OREN, D.C. 1996. Application of parsimony analysis of endemicity (PAE) in Amazonian biogeography: an example with primates. Biological Journal of the Linnean Society 59(4):427-437.

SILVA, J.M.C., SOUZA, M.C. \& CASTELLETI, C.H.M. 2004. Areas of endemism for passerine birds in the Atlantic forest, South America. Global Ecol. Biogeogr. 13(1):85-92.

SIGRIST, M.S. \& CARVALHO, C.J.B. 2008. Detection of areas of endemism on two spatial scales using Parsimony Analysis of Endemicity (PAE): the Neotropical region and the Atlantic Forest. Biota Neotropica 8(4):33-42.

SOEST, R.W.M. \& HADJU, E. 1997. Marine area relationships form twenty sponge phylogenies: a comparison of methods and coding strategies. Cladistics 13(1):1-20.

TAKIYA, D.M. \& MEJDALANI, G. 2004. Taxonomic revision and phylogenetic analysis of the sharpshooter genus Balacha Melichar (Hemiptera: Cicadellidae: Cicadellini). Systematic Entomology 29(1):69-99.

VANZOLINI, P.E. 1992. Paleoclimas e especiação em animais da América do Sul tropical. Estudos Avançados 6(15):41-65.

VAN VELLER, M.G.P., KORNET, D.J. \& ZANDEE, m. 2002. A posteriori and a priori methodologies for testing hypothesis of causal processes in Vicariance Biogeography. Cladistics 18(2):207-217.

WILEY, E.O. 1988. Parsimony Analysis and Vicariance Biogeography. Syst. Zool. 37(7): 271-290.

Received: 04/03/09

Revised: 28/10/09

Accepted: 17/11/09 